\title{
Wendelstein 7-X Torus Hall layout and System Integration
}

\author{
D. A. Hartmann, R. Krampitz, C. Damiani, U. Neuner, F. Schauer \\ Max-Planck Institut für Plasmaphysik, EURATOM Association, \\ Teilinstitut Greifswald, Wendelsteinstrasse 1, 18491 Greifswald, Germany,
}

\begin{abstract}
Wendelstein 7-X (W7-X) is an experimental magnetic fusion device presently under construction in Greifswald, Germany, to study the stellarator concept at reactor relevant parameters und steady-state conditions. It is located nearly in the center of a $30 \mathrm{~m} \mathrm{x} 30$ $\mathrm{m} \times 24 \mathrm{~m}$ hall. A large number of components need to be placed in close proximity of the torus to provide the system with the required means, e.g. cryogenic gases, cooling water, electricity, and to integrate it with the peripheral diagnostic and heating components. The arrangement of these components has to be supported by suitable structures, and has to be optimized to allow for installation, maintenance, and repair. In addition, space has to be provided for escape routes and for sufficient distance between components that could negatively influence each other's performance, etc. This paper gives an overview over the guiding principles and used tools for optimizing the layout of all components in the torus hall.
\end{abstract}

\section{Introduction}

Wendelstein 7-X is an experimental fusion device presently under construction in Greifswald, Germany, to study the stellarator concept at reactor relevant parameters und steady-state conditions. The basic machine consists of the torus that houses the magnet system and support structures, the plasma vessel and the cryostat. The magnet system is composed of 70 superconducting field coils of 7 different types that are arranged in 5 identical modules. This five-fold symmetry is only approximately retained in the openings for supply lines into the cryostat and the plasma vessel due to varying port size requirements of the diagnostics and the heating systems. A large number of components need to be placed in close proximity of the torus to provide the system with the required means, e.g. liquid helium and nitrogen, cooling water, electricity, and to integrate it with the diagnostic and heating systems. These components have to be supported by suitable structures, and have to be optimized to allow for installation, maintenance, in-service inspection, and repair. In addition, space has to be provided for escape routes and distances have to be kept between components that could negatively influence each other's performance.

The layout of the components in the torus hall has been developed over many years using 3D CAD software. It was based on simple geometric models of the components and of required surrounding space. Presently the layout design is being refined and updated by replacing the original coarse models with more refined estimates or - in some cases - with as-built models. All interface requirements are carefully taken into account. In addition, design integration tasks have been started that involve pipe routings, cable trays and support structures. 
The torus hall has the approximate dimensions of a cube of $34 \mathrm{~m} \times 34 \mathrm{~m}$ x 24 m (Fig. 1); about one third of it is below ground level. The walls are made of $1.8 \mathrm{~m}$ thick boron concrete. About $4 \mathrm{~m}$ below the ground level an intermediate concrete level with a near centric cylindrical hole of $4 \mathrm{~m}$ diameter has been added. The lowest floor in the torus hall is called "second basement", the floor of the intermediate concrete level is called "first basement". Main access to the torus hall is given via two large sliding concrete doors; the large one on the ground floor has the dimensions $8 \mathrm{~m} \times 10 \mathrm{~m}$, the smaller door on the first basement has the dimensions $4 \mathrm{~m} \times 5 \mathrm{~m}$. Additional personnel access is provided via two labyrinths on diagonally opposite sides of the torus hall. Four ducts lead to the torus hall on the level of the second basement for ECRH, ICRH, general supplies (cooling water, cryo gases) and for diagnostic cables. In addition, a small number of holes will be drilled into the torus hall walls for additional cables, gas lines etc. The air conditioning system of the torus hall provides $196 \mathrm{~kW}$ of cooling power.

The W7-X torus, also called "basic machine", includes the plasma vessel and its inside plasma-facing components and diagnostics, and the surrounding cryostat-vessel with the super conducting coil system, the coil support structure and the ports [1]. The torus has a major radius of about $5.5 \mathrm{~m}$ and a minor radius of $2 \mathrm{~m}$. It is located nearly in the center of the torus hall and is supported by a pentagon-shaped, five legged stainless steel machine base in such a way that after installation the horizontal midplane of the torus is about $1 \mathrm{~m}$ above the ground floor, and that the axis of the torus is aligned with the axis of the cylindrical hole in the floor of the first basement. The legs of the machine base are extended into the second basement so that the whole weight of the torus is supported by the floor of the torus hall.

The device has to be integrated with the plasma heating and diagnostic components and all the auxiliary components supplying the means required for operation (electricity, cryogenic fluids, cooling water, vacuum, pressurized gases). These components require access either to the plasma vessel or to the cryostat. Altogether, there are about 300 ports to the plasma vessel and about 250 openings into the cryostat.

The paper summarizes the present state of the component layout in the torus hall, and how the peripheral supply systems, diagnostics, and heating systems are being integrated into the machine. Section 2 states the principles of the layout, Section 3 describes the tools used and developed to apply these principles to the layout in the torus hall, Section 4 gives examples for the layout of the main components in the torus hall and Section 5 summarizes the present state and provides an outlook of the forthcoming tasks.

D. A. Hartmann et al., „Wendelstein 7-X Torus Hall Layout and System Integration“ Page 2 of 7 


\section{Principles of the layout}

It is necessary to coordinate the efforts to integrate the W7-X components in the torus hall with the necessary auxiliaries in such a way that:

- optimal use is made of the limited space available in the vicinity of the torus,

- all components can function properly and reliably: e.g. they are supplied with all the auxiliaries as specified, thermal and electric losses are small, the data signal-tonoise ratio is sufficiently large, the levels of magnetic field strength or radiation at the location of the components are below acceptable limits, the supports of the components are sufficiently stiff and do not transmit unacceptable vibrations from other components,

- all modes of operation of the device and the components are possible (e.g. certain components can be cooled even though the experiment is being baked)

- maximum experimental flexibility is ensured (e.g. water routing is installed so as to operate the target elements of a modified divertor with an even larger throughput of cooling water than realized for initial operation),

- all safety regulations are met (e.g. required escape routes are available, actions required in case of component failures can be performed),

- sufficient access is provided for installation, operation, maintenance, repair, removal of components, in particular of those components that are not installed at the start of operation and have to be installed later,

- the down-time of the experiment can be kept short in case of most component failures (e.g. maintenance-heavy components are placed outside the torus hall)

- future developments of the diagnostics and heating systems are possible,

- start of operation is possible soon after finishing the assembly of the basic device.

In most cases those responsible for a component take care that all these aspects are properly considered. They are supported by the two teams: System Coordination and System Integration.

System Coordination (a department within Project Coordination) collects, specifies, monitors and controls various interfaces (electrical, mechanical, etc.) between components. This is done by documenting all interfaces that two components have with each other, e.g. the target elements of the divertor and the target cooling water circuit.

System Integration (a department within System Engineering) manages additional documents that focus on the geometrical interfaces of all components outside the torus and in the torus hall, or in the hallways of buildings adjacent to the torus hall. These documents collect information on

- the location, approximate size, weight of the components,

- additionally needed assembly or maintenance space,

- available or needed support structures,

- requirements on the location, e.g. mechanical isolation, maximum ambient magnetic field,

- the planned time of installation,

- other related components.

These documents are also used for properly keeping track of new or modified space requests of the components. Therefore they are called "space-request" documents. Geometrical interfaces of components inside of the torus, in particular, collision checks D. A. Hartmann et al., „Wendelstein 7-X Torus Hall Layout and System Integration“ Page 3 of 7 
of the various components that deform under operation scenarios like cool-down, coil excitation etc. are being handled by a separate group of designers within the design office.

\section{Managing the layout in the torus hall}

The layout of the components in the torus hall is accomplished with the help of 3D CAD models realized in CADDS5, and on the basis of the space-request documents.

The documents of the components in the torus hall are labeled according to a letter key system adopted from the one used for nuclear power plants, called KKS. In general, a component family is labeled with a three letter key. These key and subsequent additional letters and numbers form the basis of the labeling also of the CAD models. According to the suffix the CAD models are additionally distinguished in part models, assembly space models, hull models etc.:

- Part models of a component are detailed models that accurately describe a part of the component. They are much too detailed to be of use for layout studies.

- Hull models of a component are solids whose outer surface is a simplified envelope over all the surfaces of the part models that make up that component but retain vital geometrical interfaces, e.g. flanges.

- Assembly space models are solids that describe the volume that is required for maintenance, assembly or installation activities. Due to shortcomings in the visualization and collision check capabilities of CADDS5 these models also have superposed a dense wireframe structure, so that during visualization one can easily toggle between these two display modes.

On various levels of detail the part models, assembly space models and hull models are arranged in assemblies. Since hull models and assembly space models are the main models used for layout control, care has to be taken to sufficiently reduce the complexity of the underlying arrangement of part models so as to keep the computer response time short during manipulation.

The location and volume occupied by the components and the additionally required assembly space are properly documented only via the CAD model. The KKS key of the diagnostic components provides some additional indication of the area within the torus hall where the component is located. Thus collision checks between various components have to be conducted by using the CAD system. Since it is impossible to perform an automatic collision check with all components within the torus hall, sub assemblies are being generated for certain areas. These assemblies contain all components within this area and automatically call upon the latest version of the models. This way, it is ensured that designers that perform the collision check are alerted of all possible neighbors within the region of interest, and new designers can easily be incorporated in the team.

During many years coarse hull models have been generated and placed in the torus hall to reserve space for the various components. This reservation was done under the KKS nomenclature of general space requests. Presently the hull models are being revised, simplified and updated for the latest design requests and stored under the proper component key. In addition, as-built models are being generated for the components in the second basement that have already been installed. The accuracy of the as-built models is typically $\pm 50 \mathrm{~mm}$ on those outermost surfaces which might become a 
geometrical interface to another component. The suppliers of components in the torus hall have been requested to provide as-built models that can be implemented in the CADDS5 system.

System Integration manages the moderation and arbitration of collisions detected between components. The person responsible for a component has to provide the properly located component hull and assembly models which form the basis of these collision checks at the time of submitting the space request document. If collisions are detected, System Integration organizes discussions between the affected parties, and provides additional input or requirements derived from other guiding principles that might pertain, i.e. from System Coordination. Non-resolvable controversies are eventually delegated to the technical director of the W7-X project. General "System Layout and Integration Meetings" are organized every two months where the outcome of these discussions are presented to a wider audience as a safeguard that all possible aspects have been considered.

\section{Layout of the main components}

The location of the main systems was decided on the basis of a trade-off between various demands: close proximity to the torus in order to reduce electrical and thermal losses and for a sufficiently high signal-to-noise ratio, a small number of openings in the torus walls, easy maintenance and repair, acceptable interaction with other components, sufficiently low levels of magnetic field or radiation, needed space and other commodities. In addition, most of the auxiliary components, e.g. power supplies, discharge resistors, were planned to be placed in the second basement, whereas diagnostic and plasma heating components were planned to be placed in the first basement or above. Many of these decisions were taken in the initial planning of the device and not yet strictly according to the principles outlined above. Eventually this led to the following general layout:

The magnet coil power supplies for the superconducting coils are located in the torus hall (second basement) in order to reduce the dissipation losses [2]. Coils of the same type are electrically connected in series inside the cryostat using a superconducting bus system. Outside the cryostat they are connected to the power supplies using normal conducting, water cooled aluminium bars. Seven power supplies are installed to independently operate the seven types of nonplanar and planar coils. Quench detection is made on the basis of measuring the voltage increase between various locations of the coils and the bus system; the location of the required electronic racks is on the first basement and as close as possible to the torus. In case of a quench the energy stored in the magnetic field is dissipated in a set of discharge resistors. The required protection circuits and the resistors themselves are located in the second basement.

The cryogenic supply system is located in a building adjacent to the torus hall [3]. The cryogenic fluids are routed into the second basement to valve boxes for the superconducting coils and the divertor cryo pump via transfer lines.

The main cooling water system will consist of separate (primary) cooling circuits for divertor and plasma vessel wall lining elements, and for the plasma vessel and port walls, heating systems and diagnostics. These cooling circuits consist of closed loops that are cooled via heat exchangers, which on the secondary side use cold water from a 
$1200 \mathrm{~m}^{3}$ large water basin. The secondary cooling circuits and pumps of the primary cooling circuit are located outside the torus hall.

Plasma heating of W7-X is provided via ECRH [4], NBI and ICRH. The 10 ECRH gyrotrons are located outside the torus hall. The high power radiation is optically directed into the second basement of the torus hall via the ECRH duct. From there additional mirrors direct the beams inside RF tight towers via RF windows into the plasma vessel. The ICRH generators are also located outside the torus hall. The coaxial transmission lines are routed via the ICRH duct into the second basement and from there to the dedicated plasma vessel ports. The NBI boxes have to be close to the vessel because of the required straight sightlines and thus are located inside the torus hall.

The various diagnostics and fuelling systems are placed within the torus hall or in adjacent buildings. In particular, lasers and microwave related diagnostics are outside of the torus hall; thus a small number of holes have to be drilled into the torus hall walls to reduce the required number of mirrors, miter bends etc.

Electronic racks needed for machine instrumentation, instrumentation and control of the various peripheral, heating and diagnostic systems are partially placed inside the torus hall. For those racks that do not have to be very close to the torus a rack shelf with three floors will be installed on the East wall that can accommodate up to 90 racks with a width of $900 \mathrm{~mm}$ each.

The present state of the installation of these components is the following: the main and the auxiliary trim coil power supplies have been installed in the second basement together with all related electronic racks and the discharge resistors. In addition, some cable trays and a section of the diagnostic secondary cooling circuit have been installed. The machine base has been ordered and will be installed at the end of 2006; the rack shelf is about to be procured. The layout of the cooling water circuits in the second basement has been planned, collisions have been eliminated and the design has been finalized. The procurement process has started and installation is planned for 2007. For illustration, Fig. 2 shows the second basement of the torus hall with the already installed and planned components. Fig. 3 provides a view of the torus hall with all the planned components; however, the support structures are still 
missing.

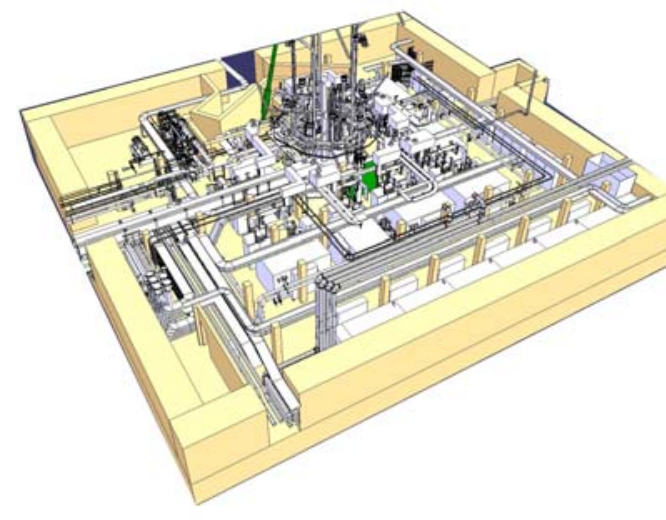

Figure 2. CAD view of the planned and partially installed components

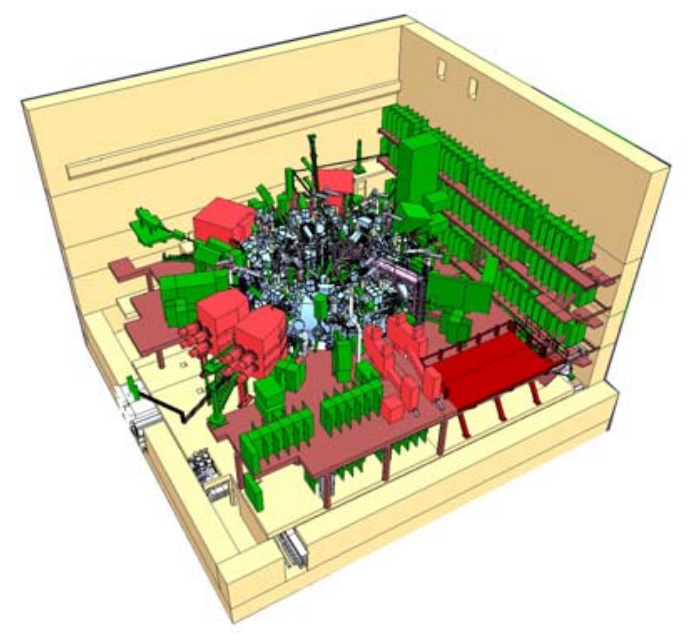

Figure 3. CAD view of the upper torus hall with simplified torus, heating and diagnostic components.

\section{Conclusion and outlook}

The tools to properly document and coordinate the arrangement of the components peripheral to the Wendelstein 7-X basic machine in the torus hall and in the adjacent buildings have been implemented. They consist of a documentation system that tracks the requirements of components and of interfaces between components. The components themselves are modelled in a 3D CADDS system. Collision checks are made based on this model. Presently the original models that were generated during the early design of Wendelstein 7-X are being updated and replaced with as-built models as far as possible. Forthcoming activities are the design of the routing of all the supply lines and cable trays, particularly in the center of the torus, the support structures of the diagnostic components taking into account the assembly and maintenance schemes.

\section{References}

[1] F. Schauer et al., Status of Wendelstein 7-X Construction, paper presented at this symposium.

[2] T. Rummel et al. "Power Supplies for the W7-X Stellarator”, Fus. Eng. Des. 6668 (2003) 1115-1118.

[3] F. Schauer et al “Cryotechnology for W7-X”, Fus. Eng. Des. 66-68 (2003) 10451048.

[4] V. Erckmann et al. "The $10 \mathrm{MW}$ ECRH and CD System for W7-X: Status and first Tests”, $31^{\text {st }}$ EPS Conf. Plas. Phys., London 2004, (Eds.) P.Norreys, H. Hutchinson. ECA 28G, EPS, Geneva (2004), P-1.197. 